\title{
Association of Mechanical Low Back Pain and Quality of Life in Security Guards
}

\author{
Mansi Manoj Mulye ${ }^{1}$, Hally Shah ${ }^{2}$, Asmaa Shaikh ${ }^{2}$ \\ ${ }^{1}$ Assistant Professor, School of Occupational Therapy, D.Y. Patil University, Nerul, Navi Mumbai. \\ ${ }^{2}$ Bachelor of Occupational Therapy, D.Y. Patil University, Nerul, Navi Mumbai.
}

Corresponding Author: Mansi Manoj Mulye

\begin{abstract}
Musculoskeletal Pain affects the bones \& soft tissue musculatures. It can be acute or chronic. It can be localized or widespread. Lower back pain (LBP) is the most common type of musculoskeletal pain. It is one of the causes of absenteeism of employees from their work and significantly affecting their quality of life (QoL). Security guards usually involve standing for long duration. Therefore LBP is quite common in them. Changes in posture or poor body mechanics may bring about spine related problems, therefore causing other muscles to be misused and become painful. Very few literatures are available worldwide on prevalence of mechanical LBP \& its relation to the QoL in standing workers. Therefore, the aim is to find out the correlation of mechanical LBP and QoL in security guards. Cross sectional study design was chosen for the research. From ninety security guards (male $\&$ female, aged 30-50 years) were screened on Cornell Musculoskeletal Discomfort Questionnaire (CMDQ). Thirtyone security guards were selected after screening, those who met the inclusion criteria (1.5 and above on CMDQ for lower back), informed consent was taken and further assessed on Oswestry LBP Disability Questionnaire (OLBPDQ) and World Health Organization QoL-BREF (WHO-QoLBREF). In the study, moderately negative correlation was found between the OLBPDQ score \& all QoL domains $(-0.3,-0.1,-0.5,-0.2)$, which showed a significant lower QoL domains with severe LBP.
\end{abstract}

Keywords: Mechanical Lower back pain, Standing workers, Quality of life

\section{INTRODUCTION}

Low Back Pain (LBP) is defined as pain and discomfort located below the coastal margin and above the inferior gluteus folds, with or without referred back pain. It is a major burden on individuals, health systems and social care system with the indirect cost being predominant. From adolescence to adulthood $80-85 \%$ of people suffer from this ailment in the modern world. Conditions in the U.S in terms of mortality or poor health as a result of disease put low back pain in sixth place, in 2010 low back pain jumped to third place. ${ }^{1}$ LBP may be classified into mechanical and non mechanical. Mechanical diagnosis of chronic low back is most common with a prevalence of $90-95 \%$ in the early phase of chronic condition (Borenstein, 1996). ${ }^{2}$

Work related LBP is any back pain originating in the context of work and considered clinically to have been probably caused, at least in part, or exacerbated by the claimant's job. It is associated with exposure to ergonomic stressors at work, environmental (physical), and psychosocial and/or personal risk factors. In many studies, a wide range of factors associated with LBP has been identified. The magnitude of the burden from low back pain has grown worst in recent years. Among these; lifting and carrying heavy objects, awkward posture, psychosocial job demands and job dissatisfaction, repetitive 
movement, static workload, bed making were reported as a major risk factor for LBP. Other factors such as sleep problem, not doing regular physical exercise, dissatisfaction with working environment and culture, duration of employment, pulling and pushing heavy load, bending and working with twisted trunks, alcohol consumption and lack of rest were also noted to be predictors of LBP. ${ }^{1}$ It is one of the causes of absenteeism of employees from their work and significantly affecting their quality of life. ${ }^{1}$

WHO defines Quality of Life as individual's perception of their position in life in the context of the culture and value systems in which they live and in relation to their goals, expectations, standards and concerns. There are major differences between men and women in the prevalence and severity of self-reported pain in the population. Quality of Life differs by gender and type of pain condition. ${ }^{3}$

\section{METHODS}

A Cross sectional study design was adopted using Systematic random sampling to study the pain intensity and quality of life (QOL) in private university. The study was conducted on security guards with mechanical low back pain. Duration of study was from September 2019 to February 2020. Subjects were selected from the 90 security guards, 31 subjects meets inclusion criterion were included in the study. Informed consent was taken for screening in a language best understood to them.

\section{Inclusion Criteria and Exclusion} Criteria: Security guards (Male and female) with mechanical LBP; who scored $1.5 \&$ more on Cornell Musculoskeletal Discomfort Questionnaire (CMDQ) for lower back and those with the age group of 30-50 years were included, whereas previous medical and surgical illness, pregnant women were excluded from the study.

\section{PROCEDURE}

Ninety security guards were taken for the study and the one whose score was 1.5 and above on CMDQ for lower back were further assessed for low back pain and quality of life. For LBP, Oswestry Low Back Pain Disability Questionnaire and for Quality of life, WHO-QoL-BREF was administered.

\section{Assessment Measure- CORNELL MUSCULOSKELETAL DISCOMFORT QUESTIONNAIRES (CMDQ):}

This questionnaire is used for the screening purpose. CMDQ has been developed by Dr. Alan Hedge and ergonomic graduate student at Cornell University. The survey is a screening tool and not a diagnostic instrument. The validity of CMDQ has been tested by Dr. Alan in English with good results. ${ }^{4}$

\section{OSWESTRY LOW BACK PAIN DISABILITY QUESTIONNAIRE (OLBPDQ):}

OLBPDQ is one of the most commonly used outcome measure for individual with low back pain. It was initially developed by John O'Brien in 1976. OLBPDQ comprises pain intensity, personal care (washing, dressing, etc.), lifting, walking, sitting, standing, sex life, social life, and travelling. ${ }^{5}$

\section{WHO-QUALITY OF LIFE- BREF \\ SCALE (WHO-QOL-BREF):}

WHO-QoL-BREF is used to assess the perception of QoL in each particular domain. QoL is defined as individuals' perceptions of their position in life in the context of the culture and value systems in which they live and in relation to their goals, expectations, standards, and concerns. The WHO-QoL-BREF is therefore based on a four domain structure (physical, psychological, social, and environmental). The WHO-QoL-BREF contains a total of 26 questions. To provide a broad and comprehensive assessment, one item from 
each of the 24 facets contained in the WHOQoL-100 has been included. In addition, two items from the overall QoL and general health facet have been included. Domain scores are scaled in a positive direction (i.e., higher scores denote higher QoL). The mean score of items within each domain is used to calculate the domain score. Then, this domain score should be converted into a transformed score to $0-100$ scale. $^{6}$

DATA ANALYSIS: The data was analysed using windows-based IBM Corp. Released 2016. IBM SPSS Statistics for Windows. Version 24.0. (Armonk;NY:IBM Corp.). Pearson correlation test were used as statistical tests of significance. The value of $P$ was set at 0.05 level of significance, and a $95 \%$ confidence interval $(\mathrm{CI})$ values were computed.

\section{RESULT}

Table 1: Correlation between Oswestry Low Back Pain Disability Questionnaire (OLBPDQ ) Score and Domains of Quality of life

\begin{tabular}{|l|l|}
\hline QoL Domain & OLBPDQ score \\
\hline Physical & $-0.3^{*}$ \\
\hline Psychological & $-0.1^{*}$ \\
\hline Social & $-0.5^{*}$ \\
\hline Environmental & $-0.2^{*}$ \\
\hline \multicolumn{2}{|c|}{ *Significant }
\end{tabular}

Table 1 shows moderately negative correlation was found between the OLBPDQ score \& all QoL domains (-0.3, $0.1,-0.5,-0.2)$, which showed a significant lower QoL domains with severe LBP.

\section{DISCUSSION}

Out of ninety subjects, 31 of them had met the inclusion criteria of the study, further assessed on OLBPDQ \& WHOQoL-BREF. Till date the prevalence of mechanical LBP and its association to the QoL in security guards has not been studied or documented. Hence, in this study, the correlation of LBP \& QoL in security guards were analyzed.

In this study, QOL for Physical, Psychological, Social \& Environmental domains scores lower with increase in LBP. This can be explained by Darzi MT et al (2014), who conducted a study on
Comparison of quality of life in low back pain patients and healthy subjects by using WHOQOL-BREF. The scores of these four domains and general quality of life and general health of WHOQOL-BREF were lower in low back pain patients. These differences were statistically significant in physical health and environmental health. ${ }^{7}$ These findings are also supported by study on Pain, kinesiophobia and quality of life in chronic low back pain and depression by Antunes RS et.al (2013), who concluded that patients with low back pain and depression had higher pain intensity, greater fear of movement and poorer quality of life. $^{8}$

\section{CONCLUSION}

The study was conducted on security guards to find out the association of mechanical low back pain and quality of life. It was found that lower back pain is a major work related musculoskeletal disorder among security guards. The standing posture of the security guards contributes to mechanical low back pain and results in poor quality of life. Appropriate preventive $\&$ intervention strategies must be emphasized to ensure a healthier \& painfree living which will ultimately improves quality of life.

Acknowledgement: None

Conflict of Interest: None

\section{Source of Funding: None}

\section{Ethical Approval: Approved}

\section{REFERENCES}

1. Sintayehu daba wami, Giziew abere, Dawit getachew, et al. Work related risk factors and the prevalence of low back pain among low wage workers: results from a cross sectional study. BMC Public Health 19, Article number: 1072(2019)[ Last accessed on 2020 march 5]

2. Kumar S., Sharma V., Shukla R., et al. Efficacy of dynamic musculostabilization techniques in the management of lumbar 
disc disorders, 2009, NJMR: Vol.14, No.1 and 2 (Issue no 22) ) [ Last accessed on 2020 march 5]

3. Sandeep Kaur, Kavita Sudhakar, Sandeep Gupta. To study the prevalence of musculoskeletal disorders in security guards. Int J Physiother. Vol 2 (6), 905-910. Available

from:https://www.researchgate.net/publicati on/285745891

_TO_STUDY_THE_PREVALENCE_OF_ MUSCULOSKELETAL_DISORDERS_IN _SECURITY_GUARDS [Last accessed on 2020, March 06]

4. CUergo: Musculoskeletal Discomfort Questionnaires; 1999. [Internet] Available from: http://www.ergo.human.cornell.edu/a hmsquest.html. [Last accessed on 2020 march 5].

5. Davidson M, Keating JL. A comparison of five low back disability questionnaires: reliability and responsiveness. Phys Ther 2002;82:8-24.

6. WHO Quality of Life-BREF (WHOQOLBREF)-Introduction, Administration,
Scoring and Generic Version of the Assessment, Field Trial Version; December, 1996.

Available

from: http://www.who.int/ mental

health/media/en/76.pdf. [Last accessed on 2020 march 5].

7. Darzi MT, Pourhadi S, et al. Comparison of quality of life in low back pain patients and healthy subjects by using WHOQOL-BREF. J Musculoskeletal Rehabilitation Volume 27, Issue 4; Pages 507-12 2014. Jan 1. [Last accessed on 2020, March 06].

8. Antunes RS, de Macedo BG, et al. Pain, kinesiophobia and quality of life in chronic low back pain and depression. Acta Ortop Bras, Volume 1, Issue 1; pages 27-9 2013, Jan 1. [Last accessed on 2020, March 06].

How to cite this article: Mulye MM, Shah H, Shaikh A. Association of mechanical low back pain and quality of life in security guards. Int $J$ Health Sci Res. 2021; 11(4): 153-156. DOI: https://doi.org/10.52403/ijhsr.20210420 